\title{
Panorama nacional do ensino da psiquiatria nas escolas médicas brasileiras*
}

\author{
An overview of the teaching of psychiatry in Brazilian medical schools
}

\author{
Alfredo Cataldo Neto ${ }^{1}$, José Cássio do Nascimento Pitta ${ }^{2}$, Marco Antônio Brasil ${ }^{3}$, Miguel Abib Adad ${ }^{4}$, Rogério \\ Wolf de Aguiar ${ }^{5}$, Camila Ruschel Selbach ${ }^{6}$, Carolina Belotto ${ }^{6}$, Cláudia Viña Coral $^{6}$, Fernanda Lia de Paula \\ Ramos $^{7}$, Patrícia Magali Simonaggio ${ }^{6}$
}

${ }^{1}$ Professor adjunto de Psiquiatria e Medicina Legal, Faculdade de Medicina, Pontifícia Universidade Católica do Rio Grande do Sul (PUCRS), Porto Alegre, RS. ${ }^{2}$ Professor assistente, Departamento de Psiquiatria, Universidade Federal de São Paulo - Escola Paulista de Medicina (UNIFESP-EPM), São Paulo, SP. ${ }^{3}$ Professor adjunto, Universidade Federal do Rio de Janeiro (UFRJ), Rio de Janeiro, RJ. ${ }^{4}$ Professor, Centro de Estudos José de Barros Falcão, Porto Alegre, RS. ${ }^{5}$ Professor adjunto, Universidade Federal do Rio Grande do Sul (UFRGS), Porto Alegre, RS. ${ }^{6}$ Médicas, PUCRS. ${ }^{7}$ Médica psiquiatra, Hospital de Clínicas de Porto Alegre (HCPA), Porto Alegre, RS.

Trabalho apresentado nos Congressos Brasileiros de Psiquiatria, na sessão oficina de ensino da psiquiatria na graduação médica, nos anos de 2008/2010.

\section{Resumo}

Objetivo: Determinar o perfil de ensino da psiquiatria nos cursos médicos brasileiros, verificando o número de professores envolvidos e suas titulações, o número de disciplinas e o enfoque predominante, material didático e local de ensino/aprendizagem.

Método: $\mathrm{O}$ estudo foi baseado no levantamento de dados obtidos pela aplicação de questionários nas escolas médicas brasileiras que constam no site da Associação Brasileira de Educação Médica.

Resultados: Das 119 escolas médicas brasileiras, 85 (71\%) responderam à pesquisa. O número de professores psiquiatras envolvidos no ensino variou de 1 a $5(75,3 \%)$; desses, 1 a $2(43,4 \%)$ eram doutores/livres-docentes, 1 a $2(45,8 \%)$ eram mestres, e 1 a $2(57,3 \%)$ eram especialistas. Observou-se que $41,2 \%$ das escolas oferecem duas disciplinas de psiquiatria, com enfoque predominantemente psicodinâmico e clínico $(61,2 \%)$. Em $52,9 \%$ dos casos, o conteúdo programático não é desenvolvido apenas em cadeiras específicas de psiquiatria. As disciplinas oferecem, em $64,7 \%$ dos casos, aulas práticas em contextos clínicos gerais. A carga horária durante o curso é de $61-90$ horas-aula (26,2\%), e 88,9\% têm livros-textos básicos. Em $83,3 \%$ das escolas médicas, essas disciplinas são avaliadas pelos alunos ao final das mesmas.

Conclusão: Foi observada uma grande heterogeneidade nas regiões avaliadas, em pelo menos $71 \%$ das escolas. Novos estudos devem aprofundar este primeiro levantamento.

Descritores: Educação, ensino, psiquiatria.

\begin{abstract}
Objective: To determine the profile of psychiatric teaching in Brazilian medical schools, with a focus on the number of professors involved and their respective degrees, the number of disciplines devoted to psychiatry and the predominant approach (psychodynamic/clinical/other) adopted, the didactic materials employed and the teaching and learning facilities available.

Methods: The study was based on the collection of data via application of questionnaires at all medical schools registered with the Brazilian Association of Medical Education (Associação Brasileira de Educação Médica).

Results: Of the 119 Brazilian schools contacted, $85(71 \%)$ returned the questionnaires. The number of professors graduated in psychiatry at each school varied from 1 to 5 (75.3\%); of these, 1 to 2 (43.4\%) had a PhD degree, 1 to 2 (45.8\%) a master's degree, and 1 to $2(57.3 \%)$ were specialists. The findings revealed that $41.2 \%$ of the schools offered two disciplines of psychiatry, with a predominantly psychodynamic/clinical approach $(61.2 \%)$. At $52.9 \%$ of the schools, teaching of psychiatric topics was not restricted to the specific disciplines; $64.7 \%$ of the disciplines offered hands-on training in general clinical settings. Mean number of credit hours of psychiatric teaching along the medical program was $61-90$ (26.2\%), and $88.9 \%$ of the programs used books as the main choice for didactic purposes. At $83.3 \%$ of the medical schools, psychiatric teaching is graded by students at the end of each discipline.

Conclusion: The present findings revealed an important heterogeneity in the geographic regions assessed, affecting at least $71 \%$ of the schools. New studies are warranted to advance the preliminary findings herein reported.
\end{abstract}

Keywords: Education, teaching, psychiatry.

* Documento da Comissão de Ensino da Psiquiatria na Graduação Médica, Associação Brasileira de Psiquiatria (ABP).

Correspondência:

Prof. Alfredo Cataldo Neto, Av. Taquara, 386/701, CEP 90460-210, Porto Alegre, RS, Brasil. Tel.: (51) 3333.8822, (51) 9956.3003. E-mail: cataldoneto@terra.com.br, cataldo@pucrs.br

Não foram declarados conflitos de interesse associados à publicação deste artigo.

Copyright (C) Revista de Psiquiatria do Rio Grande do Sul - APRS

Recebido 30/07/2008. Aceito em 20/09/2009. 


\section{Introdução}

A preocupação com o ensino da psiquiatria nas escolas médicas brasileiras vem de longa data. Em 1980, Rodrigues \& Fávero registraram que as pesquisas nessa área na América Latina, em particular no Brasil, eram escassas, havendo inclusive falta de uniformização de métodos de ensino ${ }^{1}$. De lá para cá, o panorama não mudou. Preocupados com o modelo tradicional da prática pedagógica adotada pelas escolas médicas, esses autores enfatizam que o ensino precisa considerar os aspectos referentes à formação pessoal básica do aluno, favorecendo não só aqueles que mais tarde buscarão uma especialização em psiquiatria, como também aqueles que vierem a escolher outras especialidades. Já em 1985, Kerr-Corrêa \& Silva propuseram-se a verificar a qualidade do ensino da psiquiatria no Brasil por meio da análise da qualidade, tipo e frequência de diagnósticos psiquiátricos feitos pelos residentes e internos da Faculdade de Medicina de Botucatu². Após a análise de 98 prontuários, os autores concluíram que os níveis de diagnóstico e tratamento ainda deixavam a desejar. Esse trabalho de 1985 serviu de base, na época, para melhorar o currículo do quarto ano médico, aumentando a carga horária da psiquiatria.

É opinião unânime que, para formar bons profissionais da prática terapêutica, uma adequada formação pessoal é necessária. Essa educação médica se dá em três fases distintas: a educação médica básica, a especialização e a educação continuada ${ }^{3}$.

O estudante de medicina deve, desde cedo, compreender o significado da real dimensão do conhecimento teórico e prático da psiquiatria. Como futuro médico, deve estar atento para os componentes pessoais e emocionais de qualquer problema que os pacientes apresentem em sua inter-relação com a família e a comunidade. Além disso, é essencial para sua formação que desenvolva a capacidade de avaliação e manejo dos transtornos mentais prevalentes e da relação empática com o paciente ${ }^{4}$. Dessa forma, é relevante a aplicação de um método de ensino apropriado para atingir esses objetivos.

Antes de 1960, apenas copiavam-se os modelos vigentes em países como EUA, Inglaterra, França e Alemanha. Até então, esses países ensinavam psiquiatria em grandes hospitais psiquiátricos, públicos ou privados, não possuindo programas estruturados e baseando-se apenas na relação pessoal entre professor e aluno. A partir de 1960, começaram a surgir programas de residência médica em psiquiatria no Brasil. Em 2004, para acompanhar o aumento na busca pela especialização em psiquiatria, observou-se também um cres- cimento no número de programas brasileiros de psiquiatria credenciados na Comissão Nacional de Residência Médica. Através do esforço organizacional das instituições médicas e do programa de Educação Médica Continuada da Associação Brasileira de Psiquiatria (PEC-ABP), vem-se buscando manter a qualidade do treinamento e a competência dos profissionais psiquiatras 5 .

A partir de 2003, por iniciativa de seu presidente, a ABP formalizou uma comissão constituída por cinco docentes universitários brasileiros (incluindo os autores ACN, JCNP, MAB, MAA e RWA deste trabalho) com o fim de pesquisar e elaborar um documento inédito descrevendo a situação atual do ensino da psiquiatria na graduação médica no Brasil.

Sendo assim, o objetivo deste estudo é descrever o panorama nacional do ensino da psiquiatria nos cursos médicos brasileiros, verificando o número de professores envolvidos e suas titulações, o número de disciplinas e enfoque predominante, o material didático utilizado e local de ensino/aprendizagem.

\section{Métodos}

Este estudo foi baseado em levantamento de dados obtido através da aplicação de questionários nas escolas médicas brasileiras que constam no site da Associação Brasileira de Educação Médica (ABEM) ${ }^{6}$. O contato com as instituições foi realizado via telefone, carta, e-mail e, em alguns casos, pessoalmente. Foram enviados, em seis tentativas, e-mails acompanhados de carta apresentando a pesquisa e questionário, endereçados ao diretor e/ou coordenador da psiquiatria nas diferentes escolas médicas. Nos congressos brasileiros de psiquiatria, fazia-se contato direto com os professores presentes para a busca de respostas. Os resultados foram registrados e analisados em conjunto, assim como de acordo com as regiões avaliadas (segundo classificação da ABEM).

\section{Resultados}

Das 119 escolas médicas brasileiras, $85(71 \%)$ responderam à pesquisa (Tabela 1). Foi observado predomínio de 1 a $5(75,3 \%)$ professores psiquiatras envolvidos no ensino da psiquiatria na graduação médica. Desses, 1 a $2(43,4 \%)$ eram doutores/livresdocentes, 1 a $2(45,8 \%)$ eram mestres, e 1 a $2(57,3 \%)$ eram especialistas. As Tabelas 2 e 3 apresentam os principais resultados obtidos de acordo com as regiões avaliadas.

Tabela 1 - Participação dos estados

\begin{tabular}{lcccccccc}
\hline & $\begin{array}{c}\text { Sul I } \\
\text { (RS) }\end{array}$ & $\begin{array}{c}\text { Sul II } \\
\text { (PR e SC) }\end{array}$ & SP & RJ/ES & MG & NE & N & C-O \\
\hline Total de escolas médicas & 10 & 13 & 24 & 19 & 14 & 20 & 9 & 10 \\
Número de escolas participantes & 9 & 13 & 18 & 12 & 12 & 13 & 2 & 6 \\
& $(90 \%)$ & $(100 \%)$ & $(75 \%)$ & $(63 \%)$ & $(86 \%)$ & $(65 \%)$ & $(60 \%)$ & $(60 \%)$ \\
\hline
\end{tabular}


Tabela 2 - Resultados regionais das escolas médicas em relação às disciplinas que envolvem a psiquiatria no currículo médico

\begin{tabular}{|c|c|c|c|c|c|c|c|c|}
\hline & $\begin{array}{l}\text { Sul I } \\
\text { (RS) }\end{array}$ & $\begin{array}{c}\text { Sul II } \\
(\text { PR e SC) }\end{array}$ & SP & $\mathbf{R J} / \mathbf{E S}$ & MG & NE & $\mathbf{N}$ & C-O \\
\hline $\begin{array}{l}\text { Número de disciplinas } \\
\text { que enfocam o ensino } \\
\text { da psiquiatria na } \\
\text { graduação médica }\end{array}$ & $\begin{array}{c}2 \\
(44,4 \%)\end{array}$ & $\begin{array}{c}2 \\
(46,2 \%)\end{array}$ & $\begin{array}{c}2 \\
(33,3 \%)\end{array}$ & $\begin{array}{c}\text { Empate } \\
\text { entre } 1,2 \text { e } 3 \\
(33,3 \%)\end{array}$ & $\begin{array}{c}2 \\
(50 \%)\end{array}$ & $\begin{array}{c}2 \\
(61,5 \%)\end{array}$ & $\begin{array}{c}\text { Empate } \\
\text { entre } 1,2 \text { e } 3 \\
(50 \%)\end{array}$ & $\begin{array}{c}\text { Empate } \\
\text { entre } 1,2 \text { e } 3 \\
(33,3 \%)\end{array}$ \\
\hline $\begin{array}{l}\text { Número de horas-aula } \\
\text { de ensino da psiquiatria } \\
\text { no curso médico }\end{array}$ & $\begin{array}{c}\text { Mais de } 300 \\
\text { horas-aula } \\
(33,3 \%)\end{array}$ & $\begin{array}{c}181 \text { a } 210 \\
(30,8 \%)\end{array}$ & $\begin{array}{l}91 \text { a } 120 \\
(27,8 \%)\end{array}$ & $\begin{array}{c}61 \text { a } 90 \\
(33,3 \%)\end{array}$ & $\begin{array}{l}\text { Empate entre } \\
30-60,61-90 \\
\text { e } 91-120(25 \%)\end{array}$ & $\begin{array}{c}61 \text { a } 90 \\
(50 \%)\end{array}$ & $\begin{array}{c}91 \text { a } 120 \\
(100 \%)\end{array}$ & $\begin{array}{c}61 \text { a } 90 \\
(50 \%)\end{array}$ \\
\hline $\begin{array}{l}\text { Enfoque } \\
\text { teórico } \\
\text { predominante }\end{array}$ & $\begin{array}{l}\text { Psicodinâmico } \\
\text { e clínico } \\
(100 \%)\end{array}$ & $\begin{array}{l}\text { Psicodinâmico } \\
\text { e clínico } \\
(53,8 \%)\end{array}$ & $\begin{array}{l}\text { Psicodinâmico } \\
\text { e clínico } \\
(61,1 \%)\end{array}$ & $\begin{array}{l}\text { Psicodinâmico } \\
\text { e clínico } \\
(58,3 \%)\end{array}$ & $\begin{array}{c}\text { Clínico } \\
(50 \%)\end{array}$ & $\begin{array}{l}\text { Empate entre } \\
\quad \text { clínico e } \\
\text { psicodinâmico e } \\
\text { clínico }(46,2 \%)\end{array}$ & $\begin{array}{l}\text { Psicodinâmico } \\
\text { e clínico } \\
(100 \%)\end{array}$ & $\begin{array}{l}\text { Psicodinâmico } \\
\text { e clínico } \\
(83,3 \%)\end{array}$ \\
\hline $\begin{array}{l}\text { O conteúdo programá- } \\
\text { tico é desenvolvido } \\
\text { apenas em disciplinas } \\
\text { específicas de psiquiatria }\end{array}$ & $\begin{array}{c}\text { Não } \\
(55,6 \%)\end{array}$ & $\underset{(53,8 \%)}{\operatorname{Sim}}$ & $\begin{array}{c}\text { Não } \\
(55,6 \%)\end{array}$ & $\begin{array}{c}\text { Não } \\
(58,3 \%)\end{array}$ & $\begin{array}{c}\text { Não } \\
(58,3 \%)\end{array}$ & $\underset{(61,5 \%)}{\operatorname{Sim}}$ & $\begin{array}{l}\text { Não } \\
(100 \%)\end{array}$ & $\begin{array}{l}\text { Empate } \\
\text { entre } \\
\text { sim e não } \\
(50 \%)\end{array}$ \\
\hline $\begin{array}{l}\text { As disciplinas } \\
\text { oferecem } \\
\text { aulas práticas }\end{array}$ & $\begin{array}{c}\text { Sim, em } \\
\text { contextos } \\
\text { clínicos gerais } \\
(66,7 \%)\end{array}$ & $\begin{array}{c}\text { Sim, em } \\
\text { contextos } \\
\text { clínicos gerais } \\
(69,2 \%)\end{array}$ & $\begin{array}{c}\text { Sim, em } \\
\text { contextos } \\
\text { clínicos gerais } \\
(61,1 \%)\end{array}$ & $\begin{array}{c}\text { Sim, em } \\
\text { contextos } \\
\text { clínicos gerais } \\
(50 \%)\end{array}$ & $\begin{array}{c}\text { Sim, em } \\
\text { contextos } \\
\text { clínicos gerais } \\
(75 \%)\end{array}$ & $\begin{array}{c}\text { Sim, em } \\
\text { hospitais } \\
\text { psiquiátricos } \\
(76,9 \%)\end{array}$ & $\begin{array}{c}\text { Sim, em } \\
\text { contextos } \\
\text { clínicos gerais } \\
(100 \%)\end{array}$ & $\begin{array}{c}\text { Empate entre } \\
\text { unidade de } \\
\text { internação } \\
\text { psiquiátrica em } \\
\text { hospital geral } \\
\text { e contextos } \\
\text { clínicos gerais } \\
(50 \%)\end{array}$ \\
\hline $\begin{array}{l}\text { As disciplinas } \\
\text { possuem livro-texto } \\
\text { básico }\end{array}$ & $\underset{(88,9 \%)}{\operatorname{Sim}}$ & $\underset{(84,6 \%)}{\operatorname{Sim}}$ & $\underset{(72,2 \%)}{\operatorname{Sim}}$ & $\underset{(91,7 \%)}{\operatorname{Sim}}$ & $\underset{(83,3 \%)}{\operatorname{Sim}}$ & $\underset{(84,6 \%)}{\operatorname{Sim}}$ & $\begin{array}{c}\operatorname{Sim} \\
(100 \%)\end{array}$ & $\underset{(83,3 \%)}{\operatorname{Sim}}$ \\
\hline $\begin{array}{l}\text { As disciplinas são } \\
\text { avaliadas pelos alunos } \\
\text { ao final das mesmas }\end{array}$ & $\begin{array}{c}\operatorname{Sim} \\
(77,8 \%)\end{array}$ & $\underset{(69,2 \%)}{\operatorname{Sim}}$ & $\underset{(94,4 \%)}{\operatorname{Sim}}$ & $\begin{array}{c}\operatorname{Sim} \\
(90,9 \%)\end{array}$ & $\begin{array}{c}\text { Sim } \\
(91,7 \%)\end{array}$ & $\underset{(69,2 \%)}{\operatorname{Sim}}$ & $\begin{array}{c}\text { Sim } \\
(100 \%)\end{array}$ & $\underset{(83,3 \%)}{\operatorname{Sim}}$ \\
\hline
\end{tabular}

Tabela 3 - Resultados regionais em relação aos professores envolvidos com o ensino da psiquiatria no curso médico

\begin{tabular}{|c|c|c|c|c|c|c|c|c|}
\hline & $\begin{array}{l}\text { Sul I } \\
\text { (RS) }\end{array}$ & $\begin{array}{c}\text { Sul II } \\
\text { (PR e SC) }\end{array}$ & SP & RJ/ES & MG & NE & $\mathbf{N}$ & C-O \\
\hline $\begin{array}{l}\text { Número de professores } \\
\text { psiquiatras envolvidos } \\
\text { na graduação médica }\end{array}$ & $\begin{array}{c}1 \text { a } 5 \\
(44,4 \%)\end{array}$ & $\begin{array}{c}1 \text { a } 5 \\
(92,3 \%)\end{array}$ & $\begin{array}{c}1 \text { a } 5 \\
(61,1 \%)\end{array}$ & $\begin{array}{l}1 \text { a } 5 \\
(75 \%)\end{array}$ & $\begin{array}{c}1 \text { a } 5 \\
(91,7 \%)\end{array}$ & $\begin{array}{c}1 \text { a } 5 \\
(76,9 \%)\end{array}$ & $\begin{array}{c}1 \text { a } 5 \\
(100 \%)\end{array}$ & $\begin{array}{c}1 \text { a } 5 \\
(83,3 \%)\end{array}$ \\
\hline $\begin{array}{l}\text { Número de professores } \\
\text { psiquiatras doutores/ } \\
\text { livres-docentes }\end{array}$ & $\begin{array}{c}\text { Empate } \\
\text { entre } 0 \text { e } 1 \text { a } 2 \\
(33,3 \%)\end{array}$ & $\begin{array}{c}1 \mathrm{a} 2 \\
(53,8 \%)\end{array}$ & $\begin{array}{c}1 \mathrm{a} 2 \\
(38,9 \%)\end{array}$ & $\begin{array}{c}1 \mathrm{a} 2 \\
(50 \%)\end{array}$ & $\begin{array}{c}0 \\
(58,3 \%)\end{array}$ & $\begin{array}{c}1 \text { a } 2 \\
(63,6 \%)\end{array}$ & $\begin{array}{c}0 \\
(100 \%)\end{array}$ & $\begin{array}{c}0 \\
(66,7 \%)\end{array}$ \\
\hline $\begin{array}{l}\text { Número de professores } \\
\text { psiquiatras mestres }\end{array}$ & $\begin{array}{c}3 \text { a } 4 \\
(44,4 \%)\end{array}$ & $\begin{array}{c}1 \mathrm{a} 2 \\
(61,5 \%)\end{array}$ & $\begin{array}{c}1 \mathrm{a} 2 \\
(50 \%)\end{array}$ & $\begin{array}{c}1 \text { a } 2 \\
(41,7 \%)\end{array}$ & $\begin{array}{c}1 \mathrm{a} 2 \\
(58,3 \%)\end{array}$ & $\begin{array}{c}1 \mathrm{a} 2 \\
(54,5 \%)\end{array}$ & $\begin{array}{l}\text { Empate entre } \\
0 \text { e } 1 \text { a } 2(50 \%)\end{array}$ & $\begin{array}{c}0 \\
(50 \%)\end{array}$ \\
\hline $\begin{array}{l}\text { Número de } \\
\text { professores psiquiatras } \\
\text { especialistas }\end{array}$ & $\begin{array}{l}\text { Empate entre } \\
0,1 \text { a } 2 \text { e } 3 \text { a } 4 \\
\quad(33,3 \%)\end{array}$ & $\begin{array}{c}1 \mathrm{a} 2 \\
(91,7 \%)\end{array}$ & $\begin{array}{c}1 \text { a } 2 \\
(44,4 \%)\end{array}$ & $\begin{array}{c}\text { Empate } \\
\text { entre } 0 \text { e } 1 \text { a } 2 \\
(33,3 \%)\end{array}$ & $\begin{array}{c}1 \text { a } 2 \\
(75 \%)\end{array}$ & $\begin{array}{c}1 \text { a } 2 \\
(63,6 \%)\end{array}$ & $\begin{array}{c}1 \mathrm{a} 2 \\
(100 \%)\end{array}$ & $\begin{array}{c}1 \mathrm{a} 2 \\
(50 \%)\end{array}$ \\
\hline
\end{tabular}

Quase metade das escolas médicas $(41,2 \%)$ tem 2 disciplinas que enfocam o ensino da psiquiatria, sendo esse enfoque predominantemente psicodinâmico e clínico $(61,2 \%)$ (Figuras 1 e 2). Em 52,9\% dos casos, o conteúdo programático não é desenvolvido apenas em cadeiras específicas de psiquiatria. As disciplinas oferecem, em 64,7\% dos casos, aulas práticas em contextos clínicos gerais (Figura 3). A carga horária (total) dedicada ao ensino da psiquiatria durante o curso médico é de 61-90 horas-aula (26,2\%), e 88,9\% têm livros-textos básicos (Figura 4). Além disso, em 83,3\% das escolas médicas, as disciplinas são avaliadas pelos alunos ao final das mesmas. 


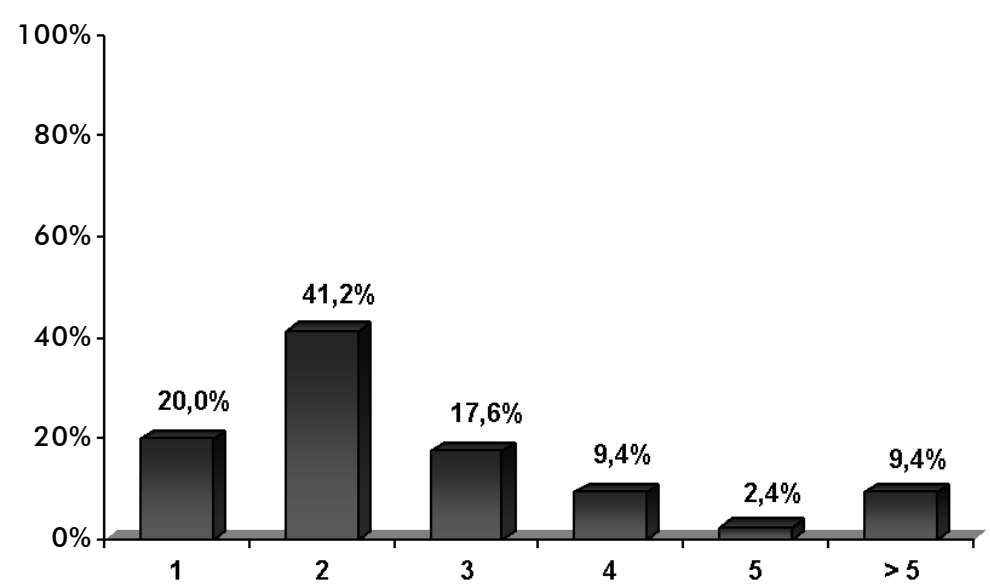

Figura 1 - Número de disciplinas que enfocam a psiquiatria na graduação médica

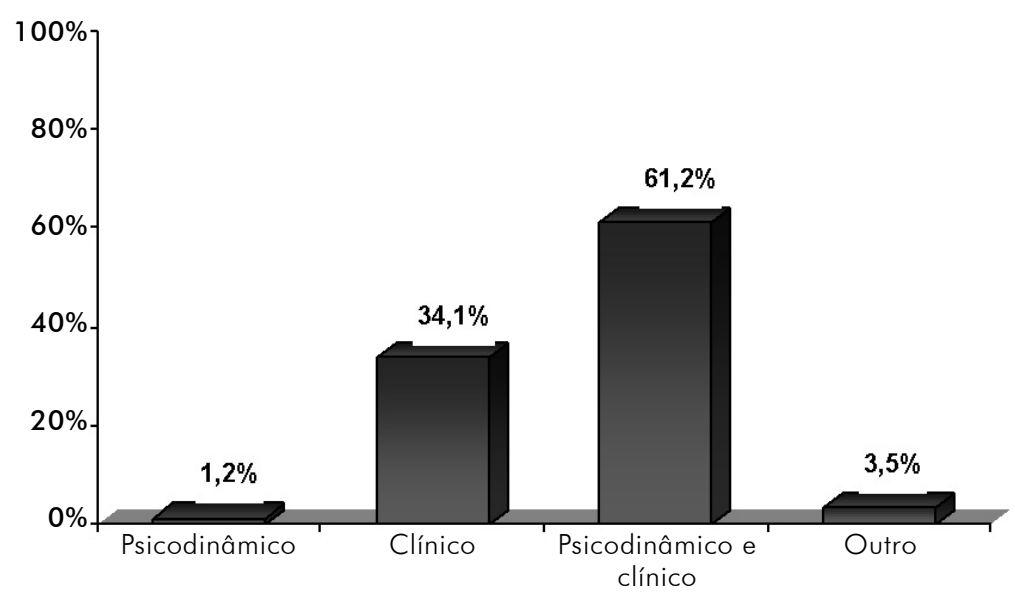

Figura 2 - Enfoque teórico predominante

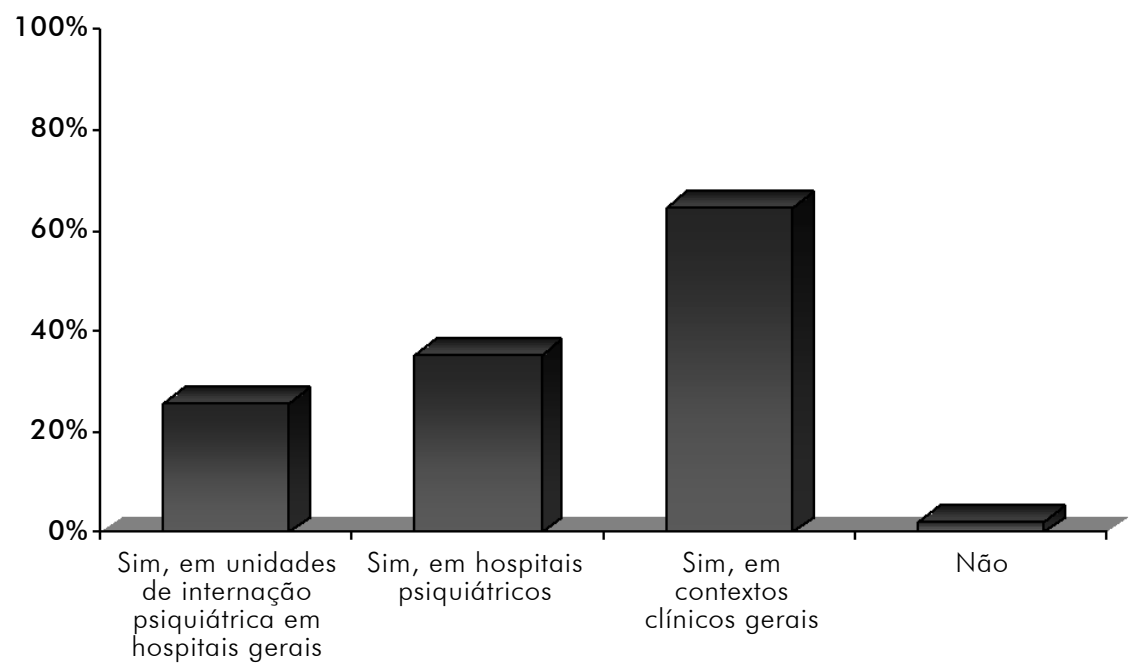

Figura 3 - Oferecimento de aulas práticas nas disciplinas 


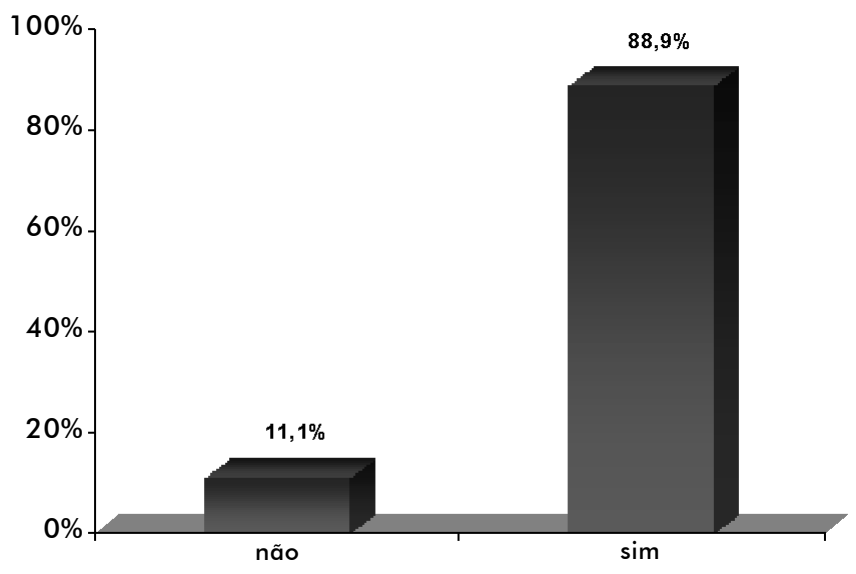

Figura 4 - Uso de livros-textos

\section{Discussão}

O presente estudo traça um panorama do ensino da psiquiatria nas escolas médicas brasileiras. Não encontramos na literatura estudo semelhante para comparações. Sendo assim, acreditamos que os resultados encontrados nesta pesquisa são válidos como uma primeira forma de levantamento.

Como vimos anteriormente ${ }^{3}$, o estudante de medicina deve receber uma preparação adequada para que seja capaz de reconhecer a doença mental, podendo, assim, efetuar um atendimento primário mais eficiente. Isso se consegue, por exemplo, aumentando a qualidade do ensino, inserindo mais conteúdos de psiquiatria no currículo médico.

As tentativas de humanizar o curso de medicina também têm sido motivo de preocupação nas instituições de ensino. Uma forma de atingir esse objetivo talvez já esteja sendo aplicada, mediante o aumento do número de disciplinas que enfocam a psiquiatria, bem como da carga horária dessas disciplinas. A psiquiatria segue sendo a guardiã do humanismo na relação médico-paciente.

Uma pesquisa de âmbito nacional como esta enfrenta a dificuldade da busca de resposta por parte dos entrevistados. Um esforço grande foi realizado nessa busca, e tivemos uma diferença muito grande entre as regiões, variando de $100 \%$ das respostas nas regiões Sul I e Sul II a $60 \%$ nas regiões Nordeste (NE) e Centro-Oeste (C-O).

$\mathrm{O}$ número de disciplinas que enfocam a psiquiatria na graduação médica mostrou um perfil predominante de duas disciplinas. Entretanto, foi observada uma grande variação, sendo que algumas escolas (especialmente nas regiões NE e C-O) possuíam apenas uma, enquanto outras chegavam a ter cinco disciplinas de psiquiatria (regiões Sul I e SP).
O número de horas-aula de psiquiatria durante o curso de medicina também variou consideravelmente nas diferentes escolas médicas brasileiras avaliadas. Enquanto que, em regiões como Sul I, as escolas apresentavam mais de 300 horas-aula ao longo do curso, outras mostravam-se incipientes na inserção da psiquiatria no currículo médico, como foi o caso das regiões Norte (N), NE e C-O.

Neste perfil, a psiquiatria no Brasil demonstrou ter um enfoque predominantemente clínico e psicodinâmico. Esse achado parece sinalizar que não se aceita mais um enfoque reducionista na psiquiatria: é preciso ensinar como identificar um conflito psíquico, mas como bem medicar uma sintomatologia psiquiátrica.

Quando perguntados se as disciplinas de psiquiatria ofereciam aulas práticas, as respostas obtidas nas diferentes regiões brasileiras foram semelhantes: todas as escolas responderam que oferecem aulas práticas e que estas se dão, em sua maioria, em contextos clínicos gerais. Além disso, a maioria das faculdades de medicina do país possui livro-texto básico para a disciplina de psiquiatria, o que mostra uma busca pela organização e pelo desenvolvimento dessa matéria no curso médico.

A formação dos profissionais envolvidos com o ensino da psiquiatria na medicina mostrou que há uma tendência à maior qualificação do ensino, já que o número de professores especialistas, mestres e doutores vem aumentando. No Brasil, temos uma média de 1 a 2 professores psiquiatras por escola, com algum tipo de formação após a residência em psiquiatria, refletindo a preocupação dessas instituições em manter a qualidade do ensino e a competência dos profissionais.

Finalmente, as disciplinas de psiquiatria estão sendo avaliadas pelos alunos ao final das mesmas na maioria das universidades, demonstrando o interesse dessas instituições em melhorar a qualidade do ensino no curso médico. 


\section{Considerações finais}

O perfil do ensino da psiquiatria nas escolas médicas brasileiras detectado por este estudo demonstra a existência de 1 a $5(75,3 \%)$ professores psiquiatras envolvidos no ensino, sendo que, destes, 1 a $2(43,4 \%)$ são doutores/livres-docentes, 1 a 2 $(45,8 \%)$ são mestres, e 1 a 2 (57,3\%) são especialistas. Verificamos, ainda, que $41,2 \%$ das escolas têm duas disciplinas de psiquiatria, com enfoque predominantemente psicodinâmico e clínico $(61,2 \%)$. Em 52,9\% dos casos, o conteúdo programático não é desenvolvido apenas em cadeiras específicas de psiquiatria, e as disciplinas oferecem, em 64,7\% dos casos, aulas práticas em contextos clínicos gerais. A carga horária predominante é de $61-90$ horas-aula $(26,2 \%)$ durante o curso, e 88,9\% das escolas têm livros-textos básicos. Em 83,3\% das escolas médicas, as disciplinas de psiquiatria são avaliadas pelos alunos ao final das mesmas.

Traçado este perfil do ensino de psiquiatria no Brasil, deparamo-nos com uma grande heterogeneidade nas regiões, em pelo menos $71 \%$ das escolas avaliadas. Novos estudos devem aprofundar este primeiro levantamento.

\section{Agradecimento}

Agradecimentos à $\mathrm{ABP}$ e às escolas médicas que gentilmente responderam às questões e permitiram, com isso, a construção deste perfil.

\section{Referências}

1. Rodrigues CRC, Fávero RV. Proposta de um modelo para a avaliação de prioridades na elaboração de currículo para o curso médico: área de psicologia médica e psiquiatria. Educ Med Salud. 1980;14(4):371-84.

2. Kerr-Corrêa F, Silva BCM. Avaliação do ensino de psiquiatria pela análise dos pedidos de interconsultas. J Bras Psiquiatr. 1985;34(4):247-52.

3. Soares CN. Perspectiva da educação continuada em psiquiatria. Rev Psiquiatr Clin. 1996/1997;23/24(4/1-3):8-17.

4. Bezerra Filho AR. A atitude de escuta na entrevista clínica: contribuição à metodologia do ensino prático da psiquiatria. Rev Psiquiatr Rio Gd Sul. 1987;9(2):120-3.

5. Camargo IB, Contel JOB. Tradução e adaptação de questionários norte-ameri canos para a avaliação de habilidades e conhecimentos na prática psiquiátrica brasileira. Rev Psiquiatr Rio Gd Sul. 2004;26(3):288-99.

6. Associação Brasileira de Educação Médica. Escolas médicas associadas à ABEM. http://www.abem-educmed.org.br/escolas_medicas.php. Acessado 20 jan 2010. 